CASE REPORTS

\title{
The carpal tunnel syndrome in congestive cardiac failure
}

\author{
A. G. ARNOLD \\ M.R.C.P. \\ North Manchester General Hospital, Delaunays Road, Crumpsall, Manchester M8 6RB.
}

\begin{abstract}
Summary
A case of the carpal tunnel syndrome is described which occurred simultaneously with congestive cardiac failure. As the cardiac failure responded to treatment the carpal tunnel syndrome improved dramatically.
\end{abstract}

\section{Introduction}

Congestive cardiac failure has not been described in association with the carpal tunnel syndrome. The author describes such a case and discusses fluid retention as a causative factor in the carpal tunnel syndrome.

\section{Case Report}

A 66-year-old man presented with a 12-month history of progressive exertional dyspnoea, paroxysmal nocturnal dyspnoea and ankle swelling. During this time he developed paraesthesiae and weakness in his hands. The weakness was so marked that he carried pliers to unzip his trousers!

On examination the patient was in gross congestive cardiac failure, with uncontrolled atrial fibrillation. He was normotensive. There was a moderately severe, bilateral median nerve motor weakness and sensory impairment, with wasting of the thenar eminences. The neurological system was otherwise intact.

\section{Investigation}

Haemoglobin $13.5 \mathrm{~g} / \mathrm{dl}$; blood sugar $7.3 \mathrm{mmol} / 1$ : free thyroxine index 72 (normal, 50-182). Serial cardiac enzymes were within normal limits. Electrocardiographs showed atrial fibrillation, widespread $\mathrm{S}-\mathrm{T}$ segment depression and $\mathrm{T}$-wave inversion, and left axis deviation. There was no evidence of left ventricular hypertrophy or of myocardial infarction. Chest X-rays revealed cardiomegaly and pulmonary oedema and X-rays of the cervical spine showed minimal spondylotic changes only.

The patient was treated with digitalis and diuretics, with an immediate, dramatic improvement of all symptoms. Three months later slight paraesthesiae remained, with no demonstrable muscular weakness. Electromyography, shortly after treatment was commenced, showed a marked delay in motor conduction in the median nerves across both wrists. When repeated 6 weeks later there was a noticeable improvement in median nerve motor conduction.

\section{Discussion}

The carpal tunnel syndrome has been described in association with many systemic diseases, including acromegaly, amyloidosis and myxoedema. It also occurs with fractures and arthritis of the wrist joint. The syndrome often occurs spontaneously, particularly in middle-aged females (Brain, Wright and Wilkinson, 1947). Pregnancy is also associated with the syndrome (Wilkinson, 1960), and fluid retention is a proposed mechanism in these cases (Guly, 1959). This aetiology might explain why diuretics are occasionally beneficial in the treatment of the disorder, although surgery is usually more effective (Brain and Walton, 1969).

The present patient developed the carpal tunnel syndrome and congestive cardiac failure simultaneously. There was no evidence that the syndrome was related to his occupation, or to other systemic disease, and it improved dramatically when his cardiac failure was treated. Although this association has not previously been described it might be expected to occur relatively frequently if the concept of fluid retention as a causative factor in the carpal tunnel syndrome is a valid one. 


\section{Acknowledgment}

I am grateful to Dr M. H. Oelbaum for permission to report this case, and the Department of Physical Medicine at Manchester Royal Infirmary for EMG studies.

\section{References}

Brain, W.R. \& WALton, J.N. (1969) Brain's Diseases of the Nervous System. 7th Edn, 781. Oxford University Press, London.
Brain, W.R., Wright, A.D. \& Wilkinson, M. (1947) Spontaneous compression of both median nerves in theD carpal tunnel. Lancet. i, 277.

Guly, P.J. (1959) Carpal tunnel syndrome. British MedicafJournal, 1, 1184.

WiLkinson, M. (1960) The carpal tunnel syndrome in pregnancy. Lancet, i, 453.

\title{
Naevus striatus unguis
}

\author{
K. LIDDELL \\ M.B., M.R.C.P. \\ M. D. Catterall \\ M.B., M.R.C.P.
}

\section{Department of Dermatology, Southampton University Hospitals}

\begin{abstract}
Summary
Three patients presented with an asymptomatic longitudinal pigmented band in a nail. A junctional melanocytic naevus in the nail matrix was suspected; this was confirmed by histological examination in each case. Differential diagnosis and management are discussed, together with a review of the literature. Local excision is considered to be the treatment of choice.
\end{abstract}

\section{Introduction}

A longitudinal pigmented band, presenting in the otherwise normal nail of a Caucasian patient is distinctly uncommon. Samman (1972) mentions the condition only briefly in Textbook of Dermatology and The Nails in Disease (Samman, 1965), and alludes to the cause as a junctional melanocytic naevus in the nail matrix. In view of its potential for malignant change, he prefers excision as the treatment of choice, after removal of the nail plate.

Pigmented bands in the nails of coloured patients are generally thought to be of less significance. The cases of two Caucasian patients who presented almost concurrently with this condition in a fingernail and a West Indian with the same clinical and histological findings in a toenail are now reported.

\section{Case histories}

\section{Case 1}

A 27-year-old Caucasian female presented with a longitudinal pigmented band in the left index fingernail (Fig. 1). It had been prominent for about 6 months, but apparently a faint yellowish browin streak had been present for some time previous There was no history of trauma and the lesion was. totally asymptomatic. Her general health was goo $\overline{4}$. She was not on any medication and there was nothing of relevance in her family or past medical history.

On examination there was a narrow, brown longi- $\stackrel{\mathbb{}}{\mathcal{Q}}$ tudinal band which appeared to emerge from the $\overrightarrow{\vec{O}}$ nail matrix and involved the full length and thickness 3 of the nail plate. No significant lymphadenopathy was detected and a diagnosis of junctional naevus of the nail of the matrix was made.

In view of the possibility of malignant change, it was decided to remove the nail plate and excise the $\frac{3}{3}$ matrix to include the suspected naevus. At operation, after avulsion of the nail, a small pigmented mark 0 corresponding to the origin of the pigmented line, was found on the matrix. This was excised with a narrow margin and the defect sutured.

Histological examination revealed the presence of a small junctional melanocytic naevus.

\section{Case 2}

A 22-year-old Caucasian naval cook presented in $N$ 1972 with a dark brown, longitudinal band in his right thumbnail. This had appeared 2 years previously, apparently spontaneously, as he had no $\mathbb{D}$ recollection of preceding trauma. A dermatologist ? made a clinical diagnosis of "junctional naevus of the $\frac{T}{0}$ nail matrix' and advised periodic follow-up, but no 